\section{PTH-136 A MYRIAD OF CAUSES FOR DIARRHOEA IN PATIENTS PRESENTING AT A GIANT (GI AND NUTRITION TEAM) CLINIC IN A CANCER CENTRE}

A Muls*, J Andreyev. GI Unit, Royal Marsden NHS Foundation Trust, London, UK

10.1136/gutjnl-2014-307263.582

Introduction Better cancer treatments have led to enormous improvements in the outcomes for these patients with the result that the overall number of survivors of cancer therapy continues to grow. However, after cancer treatment, up to $50 \%$ patients are left with diarrhoea - the most prevalent symptom. Causes are likely to encompass several contributing GI diagnoses.

Methods A service evaluation was conducted of new patients attending our clinic, reporting diarrhoea after treatment for cancer. All patients attending the clinic completed a patient recorded outcome measure describing their symptoms and a Bristol Stool Chart describing stool type. They were investigated using a peer reviewed investigational and management algorithm. Patient characteristics, symptom incidence and severity were recorded prospectively.

Results Over a 6 month period (July - Dec 2012), 207 patients were newly referred to the GIANTs. Of those, 104 (50\%) reported diarrhoea (type 6 or 7 Bristol Stool Chart). In this group there were slightly more men $(52 \%)$ than women (48\%). Their median age was 62 years (range: $22-89$ ). Primary tumour sites included urological cancer $(34 \%-82 \%$ of these prostate), gynaecological (22\%), colorectal (20\%), upper GI (10\%), haematological (8\%), and other (6\%). 69\% had undergone pelvic radiotherapy, $48 \%$ had been treated with surgery or received chemotherapy. $12 \%$ received pelvic radiotherapy alone, $6 \%$ surgery and $3 \%$ chemotherapy alone. Over a quarter (29\%) received all 3 treatment modalities.

Small intestinal bacterial overgrowth was found in $49 \%$. Bile acid malabsorption was newly diagnosed in 33\% of patients. Weak pelvic floor musculature was a contributing factor in $20 \%$. 13\% were diagnosed with new pancreatic insufficiency. Excess fibre intake $(>20 \mathrm{~g} /$ day $)$ was a contributory factor in $11 \%$ and Lansoprazole in up to $9 \%$ of patients. Other factors included: thyroid problems (9\%), anal fissure (5\%), rectal ulceration (5\%), faecal loading (5\%) and new onset Inflammatory Bowel Disease (3\%). A colorectal polyp was found in $16 \%$ of patients, 1 patient had a new colorectal cancer and 2 had a GI stricture.

$80 \%$ of patients had multiple causes for their diarrhoea. Most patients were discharged with a significant improvement in their symptoms with a median of 4 consultations (range 1-7) after systematic assessment and targeted management of the causes for their symptoms.

Conclusion Diarrhoea after cancer treatment is frequent in the patient cohort seen in our clinic. Several GI causes contribute to diarrhoea simultaneously in most patients but the majority can be discharged after a small number of consultations with a significant improvement or full resolution of their symptom if a systematic investigational and treatment approach is adopted.

Disclosure of Interest None Declared.

\section{PTH-137 THE GI AND NUTRITION TEAM SERVICE (GIANTS): MANAGING GI CONSEQUENCES OF CANCER TREATMENT - WHO? WHAT? AND HOW?}

A Muls*, J Andreyev. GI Unit, Royal Marsden NHS Foundation Trust, London, UK

10.1136/gutjnl-2014-307263.583
Introduction Our clinic specialising in gastrointestinal problems after cancer treatment, seems to be attracting patients with GI consequences resulting from a wider variety of cancer therapies. We present an analysis of new patients referred to our GI and Nutrition Team service.

Methods A service evaluation of new patients attending the clinic is ongoing after gaining appropriate approvals. All patients attending clinic complete a patient recorded outcome measure describing their symptoms, a Bristol Stool Chart describing stool type and a quality of life scale. Patients are investigated systematically depending on their symptoms, using a peer-reviewed algorithm. Patient characteristics, symptom incidence and severity are recorded prospectively.

Results Data for July-December 2012 were analysed. 207 patients were newly referred to the GIANTs. Their median age was 61.6 years (range: 22-89). 55\% were male. The largest group were patients treated for a urological malignancy (37\%), followed by those with a gynaecological (18\%), colorectal (16\%), upper GI cancer (12\%), other cancers (8\%), haematological malignancy (6\%) and no previous cancer diagnosis (3\%). $71 \%$ of patients had received pelvic radiotherapy, $3 \%$ chemotherapy, $11 \%$ GI surgery and $11 \%$ were treated with both chemotherapy and surgery. $4 \%$ had not yet received any cancer treatment and were usually referred to exclude the presence of IBD, a relative contra-indication to radiotherapy.

Comparing symptom profiles of patients who received pelvic radiotherapy $(\mathrm{n}=140)$ and those treated with other treatment modalities $(n=61)$, reveals that most patients were troubled by multiple symptoms: urgency (62 vs. 52\%), diarrhoea (57 vs. $51 \%$ ), tenesmus (47 vs. $43 \%$ ), flatulence (56 vs. $52 \%$ ), borborygmi (36\% vs $52 \%$ ) abdominal pain (39\% both groups), bloating (29 vs. $38 \%$ ), faecal leakage (16 vs. $31 \%)$ and nocturnal defaecation (31\% both groups). Rectal bleeding was reported by $34 \%$ of patients who received pelvic radiation, compared to $13 \%$ in the other group. Fatigue affected both groups (46 vs. 54\%).

The types of diagnosis to account for the symptoms made in both groups were similar: small intestinal bacterial overgrowth ( $24 \%$ pelvic radiotherapy vs. $28 \%$ other cancer treatments). Bile acid malabsorption was diagnosed in $16 \%$ (both groups) and pancreatic insufficiency in 6 and 5\%.

Conclusion Gastrointestinal problems after any cancer treatment are frequent and the symptom burden is high. The prevalence of symptoms patients describe after pelvic radiotherapy differs from those treated with other modalities but the causes identified for those symptoms are the same. A systematic management algorithm and multidisciplinary approach is required to manage those complex symptoms optimally.

Disclosure of Interest None Declared.

\section{PTH-138 TNF-A DEPENDENT ANGIOPOEITIN MEDIATED ANGIOGENESIS IN SPORADIC SMALL BOWEL ANGIODYSPLASIA; NOVEL PATHOPHYSIOLOGY AND POTENTIAL CLINICAL MARKER}

${ }^{1} G$ Holleran*, ${ }^{1 B}$ Hall, ${ }^{2} \mathrm{P}$ Crotty, ${ }^{3} \mathrm{~S}$ Smith, ${ }^{1} \mathrm{D}$ McNamara. 'Department of Clinical Medicine, Trinity College Dublin, Dublin, Ireland; ' ${ }^{2}$ Department of Histopathology, Tallaght Hospital, Tallaght, Ireland; ' ${ }^{3}$ Department of Clinical Medicine, Tallaght Hospital, Tallaght, Ireland

\subsection{6/gutjnl-2014-307263.584}

Introduction Angiodysplasias account for over 50\% of small bowel causes of obscure gastrointestinal bleeding. Angiodysplasias are thought to develop as a result of an imbalance in the 


\begin{tabular}{lllll} 
Abstract PTH-138 Table 1 & & & \\
\hline Angiogenic factor & Patient mean & Patient range & Control mean & Control range \\
\hline Ang-1 & 13071 & $615-43833$ & 21169 & $2253-56010$ \\
Ang-2 & 4600 & $842-11767$ & 2973 & $792-7995$ \\
TNF & 6.7 & $1.97-16.94$ & 12.2 & $1.73-42$ \\
Mean ratio & 1.05 & $0.06-9.26$ & 0.29 & $0.06-1.74$ \\
Ang-2/Ang-1 & & & & $<0.003$ \\
\hline
\end{tabular}

angiogenic cascade, although the exact mechanism remains elusive. Previous research we have undertaken has associated elevated serum angiopoietin-2 (Ang-2) levels with angiodysplasia. Ang-1 and Ang-2 are ligands of the endothelial receptor tyrosine kinase Tie-2. Ang-1 regulates endothelial cell survival and blood vessel maturation and plays a key role in maintaining vascular integrity. Ang-2 is a functional antagonist of Ang-1. Inflammation and angiogenesis are associated with several pathological disorders and previous data suggests a TNF- $\alpha$ dependent dual functional roles of Tie2 in inflammatory angiogenesis

Methods Following informed consent, serum samples were collected from patients with a definite diagnosis of sporadic small bowel angiodysplasia (P2) on capsule endoscopy, and from healthy controls in which GI bleeding had been out-ruled by a negative faecal immunochemical test. Serum levels of Ang-1, Ang- 2 and TNF- $\alpha$ were measured using commercially available ELISA kits. All results were expressed as a mean and compared between patients and controls, and the mean of the ratio of ang2/ang1 levels for each group was calculated.

Results A total of 80 samples were analysed for each factor, including 40 patients ( $48 \%$ male, average age 71 years) and 40 controls (43\% male, average age 70 years). As expected and in keeping with our previous work levels of Ang-2 were significantly higher in patients (mean $4600 \mathrm{pg} / \mathrm{ml}$ ) than in controls (mean $2973 \mathrm{pg} / \mathrm{ml}$ ) p < 0.001. In addition levels of Ang-1 were significantly lower in the patient group (mean $13071 \mathrm{pg} / \mathrm{ml}$ ) vs. controls (mean $21169 \mathrm{pg} / \mathrm{ml}$ ) $\mathrm{p}<0.004$ (Table 1). We also found that levels of TNF- $\alpha$ were significantly lower in the patient group (mean $6.7 \mathrm{pg} / \mathrm{ml}$ ) vs. controls (mean $12.2 \mathrm{pg} / \mathrm{ml}$ ) p $<0.003$. The mean of the ratio of Ang2/Ang1 levels was found to be significantly higher in patients (1.05) vs. controls $(0.29) \mathrm{p}<0.05$.

Conclusion Ours is the first study to have identified a link between angiopoietin 1 and 2 ratios and gastrointestinal angiodysplasia. The TNF- $\alpha$ findings are also novel and would strongly suggest a role for inflammatory mediated angiogenesis in this condition.

Disclosure of Interest None Declared.

\section{PTH-139 LONG ACTING OCTREOTIDE THERAPY HAS A BENEFICIAL EFFECT IN PATIENTS WITH SIGNIFICANT SMALL BOWEL ANGIODYSPLASIA}

${ }^{1,2} \mathrm{G}$ Holleran*, ${ }^{3} \mathrm{~B}$ Hall, ${ }^{2} \mathrm{~N}$ Breslin, 'D McNamara. 'Department of Clinical Medicine, Trinity College Dublin, Tallaght, Ireland; ' Gastroenterology, Tallaght Hospital, Tallaght, Ireland; ${ }^{3}$ Trinity College Dublin, Tallaght, Ireland

\subsection{6/gutjnl-2014-307263.585}

Introduction Angiodysplasias account for up to 50\% of causes of small bowel bleeding and due to their relative inaccessibility and the intermittent nature of their bleeding they present a particular therapeutic challenge. Endoscopic ablation with APC is the most efficacious at reducing re-bleeding rates, however; its effect is short-lived and not all small bowel lesions are amenable to treatment via DBE. It has been suggested by a recent small trial that long-acting Octreotide may be beneficial at reducing re-bleeding rates.

\begin{tabular}{|c|c|c|c|}
\hline \multicolumn{4}{|c|}{ Abstract PTH-139 Table 1} \\
\hline & Hb pre-Rx & Most recent $\mathrm{Hb}$ & Re-bleeding episodes \\
\hline Overall & $8.7 \mathrm{~g} / \mathrm{dL}$ & $11.2 \mathrm{~g} / \mathrm{dL}$ & 5 \\
\hline Octreotide only & $8.6 \mathrm{~g} / \mathrm{dL}$ & $11.1 \mathrm{~g} / \mathrm{dL}$ & 2 \\
\hline APC and Octreotide & $9 \mathrm{~g} / \mathrm{dL}$ & $11.5 \mathrm{~g} / \mathrm{dL}$ & 3 \\
\hline
\end{tabular}

Methods Following ethical approval patients with significant recurrent anaemia ( $\geq 6$ months with RCC transfusions) and definite small bowel angiodysplasia (P2), on capsule endoscopy were invited to participate in an open label, uncontrolled pilot study of monthly $20 \mathrm{mg}$ long acting Octreotide. Patients who had lesions amenable to APC via DBE were treated prior to commencing Octreotide. Baseline demographics, medications, endoscopy and capsule findings and $\mathrm{Hb}$ level were recorded. Patients were assessed at regular intervals and evaluated for side effects and episodes of bleeding (defined as either overt bleeding or a $\mathrm{Hb}$ drop of $>1 \mathrm{~g} / \mathrm{dL}$ ). Patients who received fewer than three doses were excluded from analysis.

Results A total of 22 patients, 50\% $(\mathrm{n}=11)$ female, mean age of 72 years (range 55-92) have been recruited to date, $16(73 \%)$ with isolated small bowel angiodysplasias and 6 with small bowel and colonic lesions. Of these, 17 (77\%) received at least 3 doses, $11(65 \%)$ had DBE and APC prior to commencing Octreotide, and $11(65 \%)$ were on anticoagulants (4 warfarin, 7 aspirin and 2 dual antiplatelet therapy with aspirin and clopidogrel). The mean duration of treatment was 8.1 months (range 3-15). There was a statistically significant difference in mean baseline and follow up $\mathrm{Hb}$ levels, $8.7 \mathrm{~g} / \mathrm{dL}(5-11.1)$ and $11.2 \mathrm{~g} / \mathrm{dL}(8-14.3)$ respectively, $\mathrm{p}$ $<0.001$. Overall there were 8 re-bleeding episodes in 5 patients (29\%). Prior APC treatment did not affect outcome (Table 1). Significant side effects occurred in 3 patients (13.6\%), 1 allergic skin reaction, 1 thrombocytopenia and 1 choledocholithiasis.

Conclusion Our study has shown that long acting intramuscular Octreotide is effective at improving $\mathrm{Hb}$ levels in patients with refractory small bowel angiodysplasias. A longer follow up interval will be required to determine the beneficial effect of combination therapy. We have identified a relatively high rate of serious side effects, in contrast to previous reports, which need to be taken into consideration prior to commencing treatment.

Disclosure of Interest None Declared.

\section{PTH-140 SOLUBLE PLANTAIN FIBRE INHIBITS THE EPITHELIAL ADHESION OF DIARRHOEAL PATHOGENS CLOSTRIDIUM DIFFICILE, SALMONELLA AND ENTEROTOXIGENIC E. COLI (ETEC) THROUGH INTERACTION WITH THE INTESTINAL EPITHELIUM}

${ }^{1} \mathrm{H}$ Simpson*, ${ }^{2} \AA$. V. Keita, ${ }^{2} \mathrm{JD}$ Söderholm, ${ }^{1} \mathrm{JM}$ Rhodes, 'BJ Campbell. ${ }^{1}$ Gastroenterology, University of Liverpool, Liverpool, UK; ${ }^{2}$ Clinical and Experimental Medicine, Linkoping University, Linkoping, Sweden

10.1136/gutjnl-2014-307263.586 